\title{
User-Oriented Service Guarantee Schemes for Future Optical Network
}

\author{
(Invited Paper) \\ Yuefeng Ji, Yubin Zhao, Hui Li \\ Key Laboratory of Information Photonics and Optical Communication, Ministry of Education \\ Beijing University of Posts and Telecommunications, Beijing 100876, China \\ jyf@,bupt.edu.cn, zhaoyubin2001@,gmail.com, lihui@,bupt.edu.cn
}

\begin{abstract}
Optical Network has given great contribution to the growth of telecommunication technologies. In the future, high capacity, multi service support and high quality of service or experience are the needy requirements of end users for the optical network. In this article, we reviewed promising optical network architectures such as Service-Oriented-Architecture (SOA) based Optical Distribution Network and Hybrid Wireless-Optical Broadband Access Network (WOBAN). Based on these networks, we propose several user-oriented service guarantee schemes. We deeply investigate the network performance, high bandwidth access, network protection, services mobility support, and resource allocation. All the proposed schemes are implemented on the test-beds. Experimental results indicate that these schemes could improve the service performance, and also verify their validity.
\end{abstract}

\section{INTRODUCTION}

With the enormous increase of user requirements for high capacity, more diversity and more personalization of services, the capacity and versatility of optical communication network needs to expand. In the recent years, the emerging of fast internet, high-definition video, peer-to-peer file exchange, interactive video gaming and even mobile service drive the need for bandwidth, quality of experience (QoE) guarantee, multi service support platform. Optical communication outperforms the bandwidth and power consumption of electronic solutions. Moreover, by wavelength multiplexing technologies, optical fiber is uniquely capable of integrating services with widely differing characteristics independent from each other in a single infrastructure[1]. The coming of novel solutions for fixed and mobile convergence (FMC) also brings promising solution for the optical networks, which could offer wireless mobile service for end user. All the above techniques make the optical network support multiple promising services for the increasing number of end users.

To satisfy the user requirements, this research investigates the solutions to guarantee the service performance within the architecture of next-generation optical network. Here, the future optical network should provide (a) the efficient network architecture for end user access, (b) effective resource management which could guarantee a high performance of service and QoE, (c) dedicated services with high capacity, low latency and novel service paradigms, (d) fault tolerant within the given infrastructure[2]. New user-oriented service guarantee solutions are provided based on these requirements to control and manage the optical network.

To guarantee the service performance, the optical network should have feasible architectures and intelligence management plane which could integrate with the service providers, service application systems with resource sharing and dynamic allocation, and capability for network convergence. These concepts should be implemented both in the backhaul network and access network. To achieve these goals, many researches have given great contribution for novel architectures and control schemes which could effectively provide better performance for end users. In this article, we list several schemes to illustrate the key issues for user-oriented optical network: (a) SOA based distributed optical network, (b) QoE based dynamic resource allocation scheme for optical network, (c) novel architecture for optical access in the fixed and mobile convergence field, including the protection scheme and service mobility support.

In the following contents, we will give our new achievements in the optical communication network fields, and discuss the key issues and challenges. Finally, conclude the whole article.

\section{ONGOING RESEARCH AND ACHIEVEMENTS}

\section{A. SOA-based Distributed Optical Network}

Service-Oriented-Architecture (SOA) based distributed optical networks provide not only high bandwidth connectivity but also highly dynamic operation for interconnecting distributed resources, which performs well for transfer of very large data flows between users and data centers. However, the implementation of such network has posed some challenging networking requirements that can be summarized as follows[3].

1. Creation of application to optical network interaction: 
applications need to be abstracted to a series of parameters which can be used for network configuration, while optical network has to realize resource virtualization to hide its detailed technology.

2. Co-allocation of computational resources and network resources: the distributed computing applications may well dynamically request not only high-performance computers, but also network connections, so resource co-allocation is necessary to reduce costs.

3. Advanced software environment such as application middleware, GMPLS based network control plane, resource management systems including virtualization capabilities.

4. Parallel network process at a large scale: optical network need to support establishment of parallel connections for parallelized services such as multi-CPU, multi-display visualization and so on.

5. Effective resource provisioning for large-capacity and also low-capacity connections: network resources need to provide efficient utilization and dynamic reconfiguration function in response to applications with various granularities demands.

As shown in Fig.1, the architecture extended from traditional Open Grid Services Architecture (OGSA) consists of four layers. In the application layer, both user and manager could access to network through respective portal. Service layer extended from Globus Toolkit 4 (GT4) take charge of resource management. Data Replication Service (DRS) receive user request from upper layer and work with other modules to complete resource schedule. Replica Location Service (RLS) realize the conversion from logic file name to physical site of application resource node. To transport huge amount of data, Grid File Transfer Protocol (GridFTP) modified from standardized FTP is implemented in our design. Reliable File Transfer (RFT) along with GridFTP is in charge of large file transfer. Grid Network Service (GNS) is communicated with DRS through Simple Object Access Protocol (SOAP) message, and it coordinates between the resource allocation and network control. Service layer could communicate with Generalized Multi-protocol Label Switching (GMPLS) based control layer through Grid User Network Interface (GUNI). GMPLS is implemented to collect network resource information into Resource Registry in real time manner and to control the configuration of optical resources. In resource layer, application resource nodes interconnected with network resources form distributed networks.

Based upon proposed architecture, a prototype test-bed shown in Fig. 2 has been developed. The links of data plane are shown as the real lines, and a Local Area Network (LAN) shown as dashed

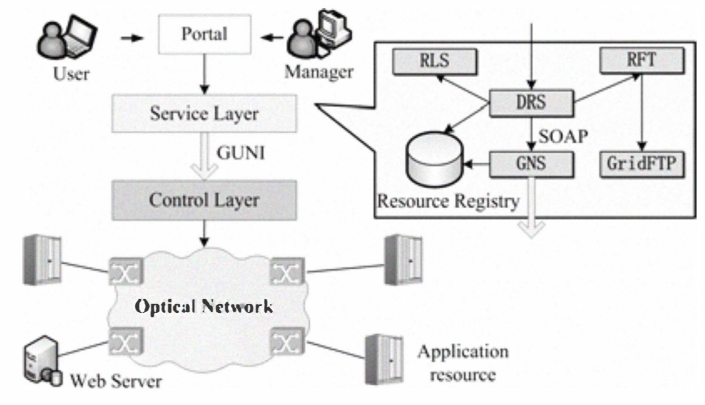

Fig.1 SOA-based distributed optical network architecture

lines constitute control plane. Application resource consists of 4 application nodes, which connect to 2 proposed Dynamic Adapter Unit (DAU) through Gigabit Ethernet (GE) link. There are four LAN ports connecting resource node and two Wide Area Network (WAN) ports connecting Optical Cross Connects (OXCs) in DAU. Network resource includes 4 GMPLS-enabled OXC and 2 GMPLS-enabled DAU interconnected by fibers. All control planes of DAU and OXC are deployed in node B. Portal service based on gridsphere locates at node A while GT 4 based service middleware locates at node B. According to Fig. 2, several experiments ground on proposed theories would be implemented on this test-bed.

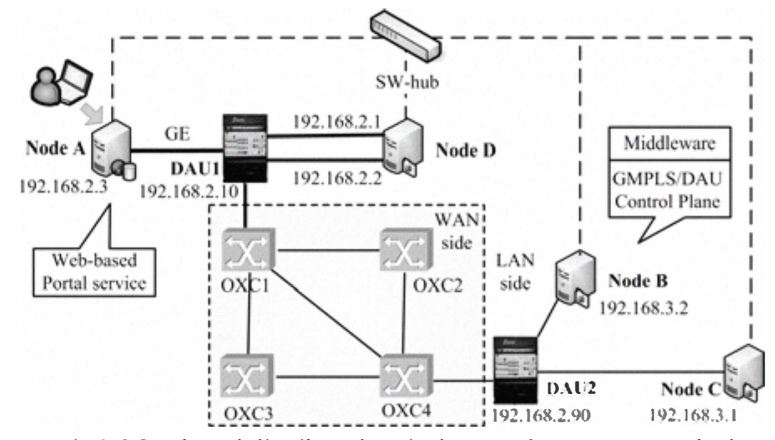

Fig.2 SOA-based distributed optical network prototype testbed

\section{B. Hybrid Wireless-Optical Broadband Access Network}

Recently, the emerging of Hybrid Optical-Wireless Broadband Access Network (WOBAN) is a promising architecture for future broadband access network. WOBAN consists of an optimal combination of an optical network as backhaul [e.g., a Passive Optical Network (PON)] and wireless access in the front-end (e.g., WiFi and/or WiMAX). The reliability and robustness of wired line optical communication and the flexibility ("anytime-anywhere" approach) and cost savings of a wireless network are integrated together in the WOBAN architecture.

Currently, a dominant broadband access technology is Passive Optical Network (PON). A PON segment starts from the telecom Center Office (CO) and consists of an Optical Line Terminal (OLT) at its head end in the CO. Each OLT can drive several 
Optical Network Units (ONU) which form the tail end of the PON. These ONUs support wireless routers in the wireless frontend of WOBAN, just as shown in Fig. 3. For the wireless access part, several architectures are proposed, which could be mainly divided into two categories. For the first kind, the wireless routers that are directly connected to the ONUs are known as wireless gateways. The wireless routers are used to efficiently manage the network connectivity in the wireless front-end. Therefore, the front end of a WOBAN is a multi-hop WMN with several wireless routers and a few gateways[4]. For the second kind, the Optical Network Unit (ONU) and Wireless Access Point (AP) are combined into a single device. Therefore, this architecture does not damage the conventional tree topology of PON, which lead a simple and cost-effective in deployment and management[5].

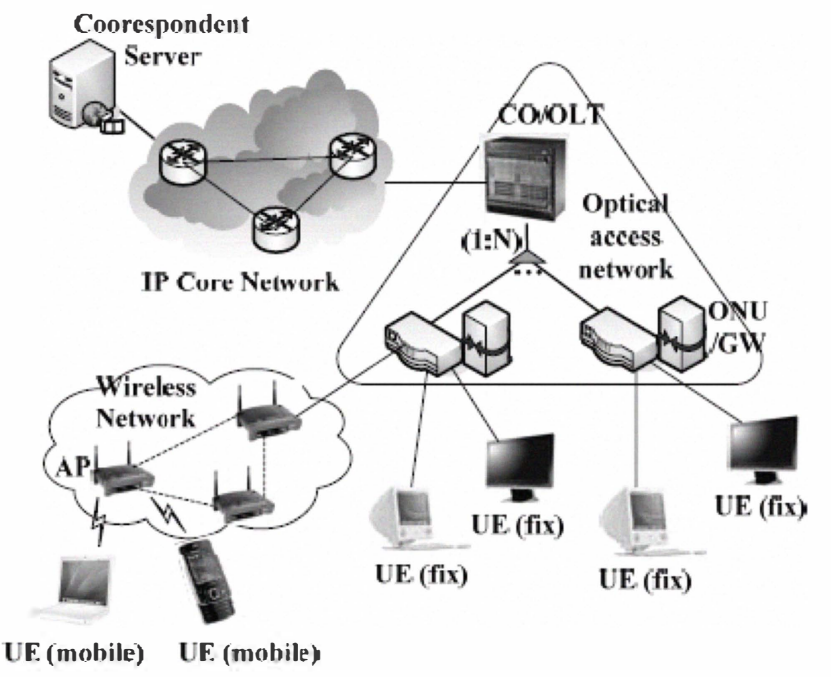

Fig. 3 Architecture of WOBAN

The advantages of a WOBAN over the wired line optical and wireless networks have made the research and deployment of this type of network more attractive. These advantages can be summarized as follows[4]:

1. Cost effectiveness: In WOBAN, we do not need expensive "fiber-to-the-home (FTTH)" connectivity, as a user will connect to its neighborhood ONU through a wireless mesh.

2. Flexibility: The wireless part of WOBAN allows the mobile users to seamlessly connect each another.

3. Robustness: In traditional PON, a fiber cut between the splitter and ONU results in an ONU failure. Furthermore, a fiber cut between OLT and splitter will disconnect a set of ONUs. However, WOBAN has the ability to reroute the traffic through the adaptive multi-hop wireless mesh in case of failures. Affected users may find a neighboring live ONU through the wireless mesh in case of an ONU failure to redirect their traffic to the CO.

4. Transport capacity: Due to its high-capacity optical trunk at the back end, the WOBAN's transport capacity is much higher than that of wireless network.

5. Reliability: WOBAN is more reliable compared to existing wireless networks as information loss due to congestion is less in WOBAN. Also, user's ability to communicate with any other ONU in its vicinity, if the primary ONU fails or is congested, gives the WOBAN a better load-balancing capability.

6. Self-organization: WOBAN which combines the PON and Wireless Mesh Network (WMN) is "self organizing" because of its fault-tolerant capability and its robustness due to adaptive wireless mesh.

To integrate the advantages of the two technologies (PON and WMN) and also avoid these drawbacks, there are still several key issues that researcher should deal with. The most important thing is to develop a flat control scheme to combine the two heterogeneous architectures. Moreover, a control bridge should also regulate network resources both in PON and WMN. Important WOBAN research issues are listed as follows:

1. Node/Router Placement: The network performance largely depends on the deployment of ONUs/gateways where the optical and wireless parts meet. Proper deployment of ONUs/gateways is critical to the cost optimization of WOBAN. However, the wireless portion is quite different from traditional architecture, since the wireless nodes can communicate with each other in a multi-hop mesh network. Each node should be able to reach AP/ONU or route the packet to the OLT/CO.

2. Routing: Once a WOBAN is deployed, routing information jointly in the wireless front-end and optical backhaul is an important problem. When the packet travels through the mesh, possibly in multiple hops, the packet delay could be significant as the packet may travel through several routers in the mesh before reaching the destination. The larger the mesh of WOBAN, the higher is the expected delay. Packet loss may also occur due to multiple scenarios. Hence, to develop a reliable efficient routing algorithm for WOBAN is an important issue.

3. Fault Tolerance: Future access networks like WOBAN should provide self-healing and self-organization properties in case of failures such as fiber cut, gateway/router failure, OLT/ONU failure, etc.

4. Topology Management: The wireless mesh front-end should be adaptive and self organizing to create a mesh topology after initial deployment or after failure.

5. Link Scheduling: Link scheduling (as a part of TDM MAC) in $\mathrm{WMN}$ is vital to increase throughput by reducing collisions. The capacity (TDM slots) of links in the WMN is carved out of the radio capacity of nodes considering interference between transmissions.

6. Joint Design: Both the PON and WMN segments in WOBAN should be designed jointly so that they complement each other. Specifically, the optical part has to be designed to help the WMN 
overcome its capacity limitation, and the wireless part has to be designed to extend the reach of PONs. Cross-layer design spanning across the physical, link, and network layers of both optical and wireless segments of WOBAN will provide better insight to enhance the performance.

In the next two sections, we will review our two research achievements in the WOBAN fields. Both of them could guarantee the service performance and offer users a better experience.

\section{Wireless Protection Switching for Optical Network}

As many of the research work generated on hybrid optical and wireless access network, wireless resources are mainly used to extend the service and area for end users. Some of them figure out that wireless network could protect the optical access network in the hybrid mode when meet any fiber link failure or ONU/OLT failure. However, none of the research ever provided effective solution by using wireless resources. Even some wireless router algorithms in the Wireless Mesh Network (WMN) could solve such problem, it will make the video service will consume huge bandwidth so that it will degrade the whole network performance. Besides, the traditional fiber protect schemes are expensive and complicated in deployment.

To protect the video service effectively, the scheme using the wireless link between the adjacent ONUs is proposed. When a fiber link failure happens, the wireless protection switching link will be aroused to reorganize the network architecture. As Fig. 4 shows, when the fiber from splitter to $\mathrm{ONU}_{1}$ is cut, the $\mathrm{ONU}_{1}$ will detect the failure and start the protection mode by searching the adjacent $\mathrm{ONU}$ such as $\mathrm{ONU}_{2}$ according to a particular protection-scheme list with sending the request signal. When $\mathrm{ONU}_{2}$ receives this request, it will make a judgment that whether it could afford the new service payload. If so, the $\mathrm{ONU}_{2}$ will set up a wireless link with ONU1, meanwhile the Centre Office (CO) will switch the data transmission of the fiber link of $\mathrm{ONU}_{2}$. However, if the $\mathrm{ONU}_{2}$ could not afford such adjacent service, $\mathrm{ONU}_{1}$ will turn to other ONUs for request. Besides, the user classified scheme is used to reduce the collision.

To evaluate the effectiveness of proposed scheme, a test-bed based on this scheme is constructed. Architecture of the test-bed is illustrated in Fig. 8: the integrated network is combined with the EPON network and wireless router (WR) with WiFi technology. Here, the new ONU is the combination of the ONU and wireless router as the border devices controlled by the Proctor. In that case,

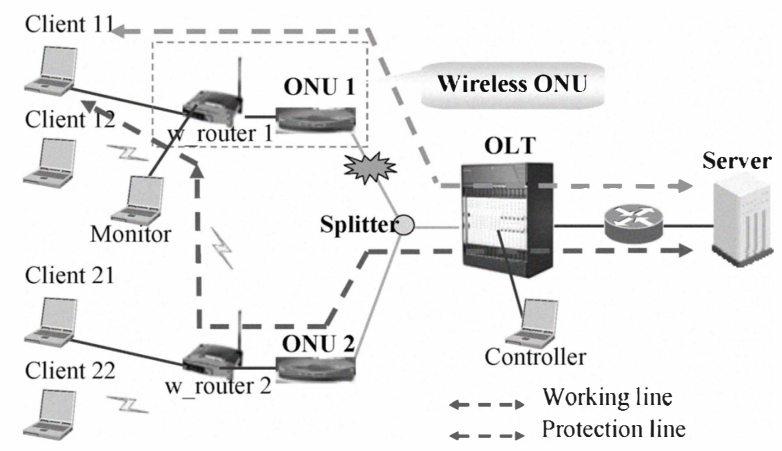

Fig. 4 Wireless Protection Switching Scheme

the ONU and wireless router (WR) could work as an integrated component as the ONU under the control of Protector. A high-performance computer provides MPEG-2 coded video service with the average the bit rates about $15 \mathrm{Mbps}$. Experimental results indicate that the protection scheme could protect the fiber link failure effectively[5].

\section{Seamless Handover Scheme in the Hybrid Architecture}

With the emergence of various access ways, the service mobility in the hybrid networks becomes to be necessary. In order to support the service mobility between the fixed terminals and the mobile terminals, we proposed that each ONU equips a gateway (GW) assisting to implement the service mobility.

There are two situations of service mobility in WOBAN: one is between different user equipments (UE) which belong to one ONU, and the other is between different user equipments belong to different ONUs. The mobility strategy is different when it comes to the different situation[6].

Considering that mobile UE_old requires to change its ongoing service to the fixed UE_new which is inspired by the user's choice or the measurement about the distance between old UE and new one. The two scenarios are both possible in the real environment, and the distance measurement can be accomplished by using the Wireless Sensor Network (WSN) or Radio Frequency Identification (RFID). These problems are not discussed in this paper. The task of this paper is seamlessly transferring the ongoing service in the UE_old to the UE_new as lower delay, and loss rate.

The GW connecting to each ONU has these central functions:

1. To identify the INITIATE message which contains the fundamental fields as the IP address of UE_old and UE_new as well as the Logic Link ID (LLID) of ONU and the port of service which wants mobility.

2. To sustain the Mobility Table which defined to map the IP address of UE_old to the IP address of UE_new for the service of certain port. For example, if the UE_old whose IP address is 192.168.0.1 wants to transfer the service identified with port 1537 
to UE_new whose IP address is 192.168.1.1, then the Mobility Table is seen in Table II.

3. To identify the service mobility ACK message which represents new GW has received the aimed service packages.

4. To filter the aimed service packages related to the certain port and to replace the destination IP address field with that of UE new.

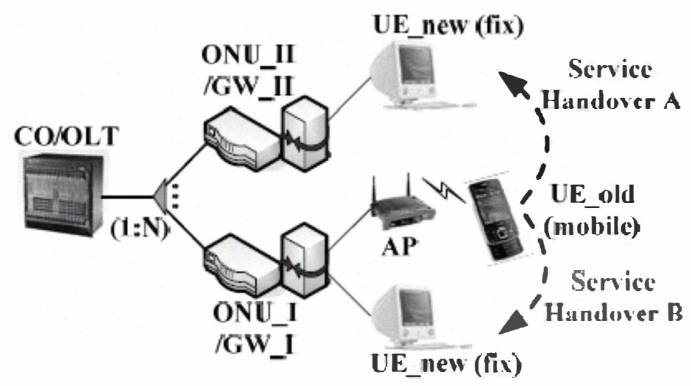

Fig. 5 Service Mobility Handover Scheme

We also implemented our scheme into a test-bed. As shown in Fig. 5, the service could follow the user at anytime and anywhere. In this test-bed, we use soft mobility strategy. The proposed strategy sufficiently utilizes the broadcast characteristic of OLT and sets LLID of ONU according to the mobility scenario. It shows high flexibility in its applicability to different mobility scenarios (under the same ONU and under the different ONUs). The given description focuses on details about the service mobility strategy which consists of two phases: service initiation phase and service execution phase. The experimental results clearly indicate that the mobility time of the strategy is short and can satisfy the requirements of multimedia users. Furthermore, the experimental results also demonstrate that the mobility strategy has little influence on the change of MLR, since the value of MLR posterior mobility stays at an acceptable level compared to that previous mobility.

\section{E. User-Classified Dynamic Resource Allocation Scheme}

VBR video traffic is expected to take a significant portion of multimedia applications for its satisfactory perceptual quality. But owing to its high traffic abruptness characteristic, bandwidth requirement of VBR video traffic undergoes both short- and long-term changes. Thus, allocating a constant amount bandwidth for VBR video stream will lead to either network resource over-allocation with low utilization or network performance degradation with delay and data loss. Therefore, network resource must be periodically renegotiated and used as minimal as possible with a given QoS. Trying to meet this challenge, the online dynamic allocations are desirable and several relevant methods have been proposed[7].

However, two problems exist in these methods. Firstly, most current solutions have not taken practical condition of network resource into account and in their study the bandwidth resource is supposed to be unlimited. So under practical multi-user condition, an adjustment collision may be produced when several users request to increase the bandwidth simultaneously while the available bandwidth resource is insufficient. Secondly, because all the prediction algorithms produce error, the allocation absolutely following prediction traffic value may be invalid and degrade the network performance. At the same time, most of the current methods use QoS parameters, such as time delay, only to certify the efficiency of the adjustment scheme while these parameters can also provide a reference for the adjustment. So an authority and helpful QoS measurement mechanism is required.

To overcome these problems, this paper proposes an integrated user-level classified dynamic resource allocation scheme to adaptively determine the bandwidth share for each user. By mastering related knowledge of the network synchronously, this scheme can effectively manage bandwidth resource with QoS guaranteed adjustment collisions avoided.

Traffic predictor provides major basis of the bandwidth adjustment and influences the performance of dynamic resource allocation enormously. In this scheme, time-domain normalized least mean square (NLMS) linear predictor performs the online real-time traffic prediction for its low computational requirement and relatively high prediction accuracy.

Experimental results show that the MDI parameters are under the recommended threshold value in most time during adjustment process and the bandwidth resource utilization is improved by $20 \%-60 \%$ compared to the traditional deterministic fixed service rate reservation. Moreover, practical multi-user application is tested and the adjustment collisions can be efficiently avoided.

To verify the effectiveness of proposed scheme, a test-bed based on this scheme is constructed. Architecture of the test-bed is illustrated in Fig.6.

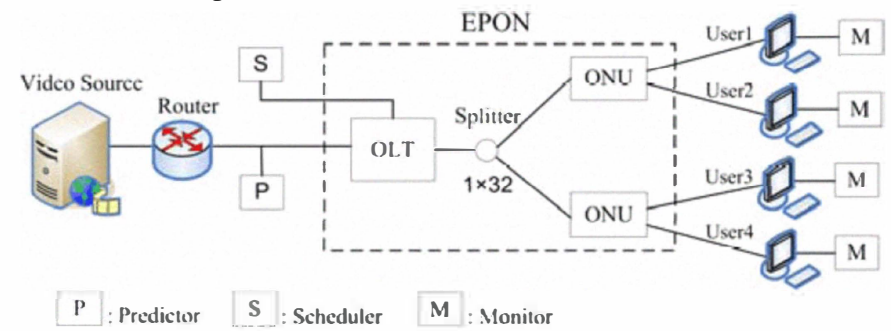

Fig. 6 Resource Allocation Scheme test-bed

As shown in diagram, a high-performance computer provides the MPEG-2 coded movie "X-man 2" as video source. Another computer performs the role of predictor by capturing the video traffic transited through router. Ethernet optical passive network (EPON) here is used as access network for broadcasting the data to the four users joining to optical network unit (ONU) and scheduler manages the bandwidth resource by writing command 
to optical line terminal (OLT). Monitors are integrated into the terminators' computers. Besides, scheduler, monitor and predictor communicate with each other using in-band TCP/IP connection.

The main advantages of the proposed scheme are:

1. To prevent adjustment collisions, practical condition of network resource is taken into account and a user-classified adjustment strategy named adjustment priorities classified strategy is put forward.

2. To avoid the resource allocation error caused by the prediction error and provide a complementary adjustment reference for the prediction results, MDI is introduced into this scheme not only as an authority real-time QoS measurement, but also as an adjustment reference.

3. Using several technologies synthetically, a practical dynamic resource allocation scheme is developed and the conception of control plane is introduced.

The experimental results obtained from test-bed clearly indicate that the VBR video transmission managed by the scheme of this paper can achieve a higher bandwidth utilization compared to the conventional fixed bit rate service model with QoS guaranteed. Furthermore, these data also demonstrates that the user-classified dynamic resource allocation scheme investigated here is feasible and flexible in practical application.

\section{CONCLUSION}

In this article, we discussed several novel network architectures and control schemes for the future optical network. The proposed architecture of SOA based Optical Distribution Network could provide the end users with multi services and intelligently control and regulate the wavelength and bandwidth which could guarantee the QoS effectively. The WOBAN architecture for future broadband access integrates the wireless technology and services with optical network. We proposed wireless protection switching scheme to protect the fiber link failure and seamless service mobility scheme to support the mobile service in this new architecture. Besides, to increase the service performance and effective bandwidth utilization, we proposed user-classified network resource allocation scheme. All proposed schemes are deployed on test-beds successfully and experiment results have verified their validity.

\section{ACKNOWLEDGMENT}

This research was supported in part by National 863 Program (No. 2009AA01A345), National 973 Program (No. 2007CB310705), NSFC (No. 60932004), P. R. China.

\section{REFERENCES}

[1] S. Figuerola and M. Lemay, "Infrastructure Services for Optical Networks [Invited]," Optical Communications and Networking, IEEE/OSA Journal of, vol. 1, pp. A247-A257, 2009.

[2] P. V. B. Primet, S. Soudan, and D. Verchere, "Virtualizing and
Scheduling Optical Network Infrastructure for Emerging IT Services [Invited]," Optical Communications and Networking, IEEE/OSA Journal of, vol. 1, pp. A121-A132, 2009.

[3] Z. W. Yuefeng Ji, Hui Li, "Design and Control of Evolving SOA-based Distributed Optical Networks," ICOCN 2009, 2009.

[4] S. D. Sarkar, "Hybrid Wireless-Optical Broadband-Access Network (WOBAN): A Review of Relevant Challenges," JOURNAL OF LIGHTWAVE TECHNOLOGY, vol. 25, 2007

[5] Y. Zhao, L. Hui, R. Xie, Y. Qiao, and Y. Ji, "Wireless protection switching for vedio service in wireless-optical broadband access network," in Broadband Network \& Multimedia Technology, 2009. IC-BNMT '09. 2nd IEEE International Conference on, 2009, pp. 760-764.

[6] Y. Z. Ruitao Xie, Danping Ren, Hui Li, Yuefeng Ji, "Demonstration of a Service Mobility Strategy in Wireless-Optical Broadband Access Networks," ICOCN 2009, 2009.

[7] X. Zhiyuan, L. Hui, L. Yueming, and J. Yuefeng, "User-Classified Dynamic Resource Allocation for Real-Time VBR Video Transmission Based on Time-Domain Traffic Prediction," in Global Telecommunications Conference, 2008. IEEE GLOBECOM 2008. IEEE, 2008, pp. 1-5. 